\title{
Outcome of Humeral Shaft Fracture Treated with Intramedullary Nail and Plate Fixation
}

\author{
Yansong Wang, Yogesh Kayastha, Yang Cao, Zhanpeng Guo, Yajiang Yuan and Yunlong BI \\ Department of Orthopaedics, First Affiliated Hospital, Jinzhou Medical University, Jinzhou, Liaoning, PR China
}

\begin{abstract}
Objective: To compare the results of the intramedullary nail (IMN) and compression plate fixation performed for humeral shaft fracture as to determine a better option out of the two.

Study Design: Comparative study.

Place and Duration of Study: First Affiliated Hospital of Jinzhou Medical University, Liaoning, PR China, from October 2016 to January 2019.

Methodology: Patients treated with IMN $(n=26)$ or plate fixation $(n=30)$ for humeral shaft fracture were included in this study. Assessment was done in terms of perioperative parameters, complications, union time, and functional outcomes. Functional outcome were compared between the two groups at each follow-up (6 weeks, 3, 6, and 12 months) and between the subsequent follow-ups in both groups using the repeated measures ANOVA.

Results: Intraoperative blood loss, operative time, hospital stay, and union time were significantly lower in the IMN group. There was no significant difference in the functional outcomes when it was compared between the two groups at each follow-up. However, when it was compared between subsequent follow-ups, a significant improvement was observed in both groups. Increase incidence of individual complication and reoperation were established in the plating group, but without a significant difference. Yet, the overall complications rate was significantly higher in the plating group.

Conclusion: IMN fixation led to a significant decrease in intraoperative blood loss, shorter operating time, hospital stay, union time, and a lower rate of overall complications. Thus, IMN may be a better choice of internal fixation as it also accelerates the patients' recovery, and increases their satisfaction.
\end{abstract}

Key Words: Humeral shaft fracture, Internal fixation, Plating, Intramedullary nails, Functional outcome.

How to cite this article: Wang Y, Kayastha Y, Cao Y, Guo Z, Yuan Y, BI Y. Outcome of humeral shaft fracture treated with intramedullary nail and plate fixation. J Coll Physicians Surg Pak 2020; 30(1):73-78.

\section{INTRODUCTION}

The conservative management of humerus shaft fracture has a union rate as high as $90 \% .{ }^{1}$ Although a complete reduction into anatomical position is rarely achieved up to $3 \mathrm{~cm}$ of shortening, less than 30 degrees of rotation and up to 20 degrees of angulations is considered acceptable. ${ }^{2}$ But nowadays, as a result of poor tolerance of the treating surgeon to the previously thought as an acceptable deformity and patient's unwillingness to go through intensive periods of conservative management, surgical treatment is highly preferred. ${ }^{3}$ Further, there are specific indications for operative management; polytrauma, obese and elderly patient with osteoporosis, segmental fracture, open fracture, radial nerve injury, associated neurovascular injuries, early unacceptable reduction or failure of conservative management, and floating elbow. ${ }^{1}$ Many randomised controlled trials have reported plate and

Correspondence to: Yang Cao, Department of Orthopaedics,

First Affiliated Hospital, Jinzhou Medical University, Jinzhou,

Liaoning, PR China

E-mail: caoyang_jzmu2017@126.com

Received: February 13, 2019; Revised: June 13, 2019;

Accepted: July 06, 2019
IMN fixation for humeral shaft fracture. Some studies showed no difference in the radiological and functional outcome of patients after the two methods. ${ }^{4}$ Moreover, there has been no consensus on the effectiveness of these two methods. Study conducted by Zarkadis et al. 5 and other colleagues demonstrated that open reduction and internal fixation (ORIF) was the preferred surgical treatment option for humeral shaft fractures in adult patients, but it requires an extensive operation with a larger incision, extraction of soft tissue and periosteum from the bone. 5 This increases the risk of radial nerve palsy, infection, delayed union and non-union. ${ }^{6}$ On the contrary, Changulani et al. concluded that IMN is a better and standard surgical method. 7

The aim of this study was to compare the outcome of IMN and compression plate fixation for treatment of humeral shaft fracture with regard to the preoperative symptom period, intraoperative blood loss, operative time, hospital stay, union time, union rate, functional outcomes, and a better overall outcome.

\section{METHODOLOGY}

This comparative study on the treatment of humeral shaft fracture using antegrade $\operatorname{IMN}(n=26)$ and 
compression plate $(n=30)$ fixation was conducted at the First Affiliated Hospital of Jinzhou Medical University from October 2016 to January 2019. The study was approved by the University ethics committee. All data were collected by the resident doctor involved in this study. Inclusion criteria were patients of age 18 years or above, closed or open humeral shaft fracture of GustiloAnderson grades I and II, fracture of $<2$ weeks duration, humeral shaft fracture treated with IMN fixation or compression plate fixation. Exclusion criteria were age $<18$ years, pathological fractures, previous humeral fracture, open fracture grade III, fracture of more than 2-week duration, the patient who lost to follow-up, and uncooperative patients due to head injuries.

Patients were chosen for the treatment after meeting the inclusion criteria, according to the fracture morphology and the decision of the treating surgeons. Informed written consent for surgery were taken in all cases with explanation of the treatment, its risk and complications.

Study design to be conducted was also explained to the patient. Preoperatively, the demographic parameters and fracture morphological characteristic were recorded. All surgeries were conducted by two surgeons familiar with both procedures. Precisely, the fractures were situated $2 \mathrm{~cm}$ distal to the surgical neck of the humerus or $3 \mathrm{~cm}$ proximal to the olecranon fossa. ${ }^{7}$ The open fracture was classified according to Gustilo-Anderson. All fracture was classified according to the $\mathrm{AO}$ classification system. In IMN group, subtypes noted were: A1 (2 cases), A2 (8), A3 (5), B1 (2), B2 (6), B3 (3). In Plating group A1 (4), A2 (9), A3 (5), B1 (3), B2 (5), B3 (2) and $C 1$ (2). In polytrauma patients, the associated injuries were pelvis fractures $(n=5)$, floating elbow $(n=3)$, clavicle fracture $(n=4)$, scapula fracture $(n=3)$, femoral shaft $(n=3)$, tibia $(n=4)$, and chest injury $(n=3)$.

Only antegrade nailing was performed in the IMN group (Figure 1). In ORIF with plate fixation, the commonly used approach was the lateral approach $(n=21)$ (Figure 2) followed by the anterolateral $(n=6)$ and then the posterior $(n=3)$. Locking compression plate (LCP) was used frequently in this study $(n=23)$ followed by limited contact dynamic compression plate (LCDCP, $n=4)$ and traditional $4.5 \mathrm{~mm}$ dynamic compression plate (DCP, $\mathrm{n}=3$ ).

A loading dose of intravenous antibiotics was administered during the induction of anesthesia and after the operation for at least three days. The preoperative symptom period, intraoperative blood loss, operative time, days of hospital stay of all the patients were recorded. A standard plain radiograph was obtained in the follow-up at 6 weeks, 3 months, 6 months and 12 months postoperatively. Functional outcome of the upper limb was assessed at each follow-up using the American Shoulder and Elbow Surgeon (ASES) scoring that incorporates the Visual Analogue Scale (VAS) score and the Activity of Daily Living (ADL) score.8,9

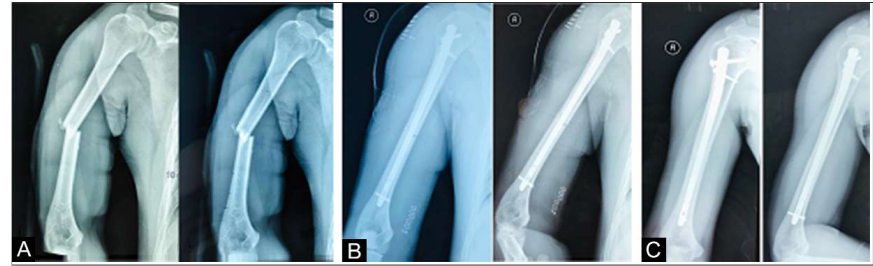

Figure 1: IMN fixation- $X$-ray of a 30 years old male with right sided humerus shaft fracture $(\mathrm{A})$, post-operative X-ray with IMN fixation (B), and X-ray at 12 months follow-up $(\mathrm{C})$.

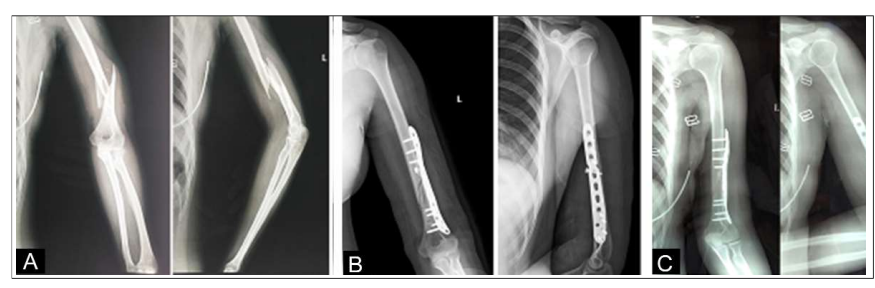

Figure 2: Plate fixation- X-ray of a 29 years old female with left sided humerus shaft Holstein Lewis fracture (A), Post-operative X-ray with plate fixation (B), and X-ray at 12 months follow-up (C).

Complications, if present, were recorded from the postoperative period until 12 months for comparison later. Union was referred to the fracture that healed within a period of 6 months.10,11 "Non-union" was considered when the fracture did not heal in a period of the first 6 months. ${ }^{11}$ These cases were followed at least another 6 months for a probable delayed union, after which the possibility of secondary treatment was considered. Therefore, delayed union was defined as subsequent healing of non-union after 6 months to one year. 10 In the plating group, two patients lost to follow-up and one case had implant failure that was managed by reoperation with plating and bone graft. Thus, the final evaluation was done in 27 cases of plating and 26 cases of nailing. The implant failure case was considered and included as a complication later on in this study.

Statistical analysis was performed using SPSS software version 22 (IL, Chicago, USA) for Windows. Fisher's exact test or Chi-square test was used to examine the relationship between qualitative variables. Continuous data with normal distribution were presented as mean \pm SD and was compared using t-tests. Repeated measure ANOVA was used to compare the overall difference in the functional outcome between the two groups at each follow-up and between the subsequent follow-ups in both groups. A p-value of less than 0.05 was considered statistically significant.

\section{RESULTS}

The mean age of the patient was $45.69 \pm 16.27$ years in the IMN group and $45.40 \pm 16.94$ years in the plating group $(p=0.948)$. The age range in IMN group and plating group were (20-79) and (18-88), respectively. In IMN group, 16 were males $(61.53 \%)$ and 10 were females $(38.24 \%)$. Whereas, in the plating group, 17 were males $(56.66 \%)$ and 13 were females $(43.33 \%)$, 
Table I: Comparison of preoperative symptom time, intraoperative blood loss, operative time, hospital stays, union time, and union rate.

\begin{tabular}{|c|c|c|c|c|}
\hline Variables & $\begin{array}{c}\text { IMN } \\
(n=26)\end{array}$ & $\begin{array}{l}\text { Plating } \\
(n=30)\end{array}$ & Statistics & p-value \\
\hline Preoperative symptom period in days (mean $\pm S D$ ) & $3.85 \pm 2.58$ & $3.27 \pm 3.11$ & $t=0.750$ & 0.457 \\
\hline Intraoperative blood loss in ML (mean $\pm S D$ ) & $61.04 \pm 6.82$ & $150.53 \pm 6.66$ & $\mathrm{t}=49.54$ & $<.001^{*}$ \\
\hline Operative time in minutes (mean $\pm S D$ ) & $67.65 \pm 4.80$ & $121.27 \pm 11.79$ & $t=22.80$ & $<.001^{*}$ \\
\hline \multirow[t]{2}{*}{ Hospital stay in days (mean $\pm S D$ ) } & $6.31 \pm 1.66$ & $12.60 \pm 2.62$ & $t=10.85$ & $<.001^{*}$ \\
\hline & $(n=25)$ & $(n=25)$ & & \\
\hline Union time in month (mean $\pm S D$ ) & $3.36 \pm 1.52$ & $4.68 \pm 2.30$ & - & $0.022^{*}$ \\
\hline Union rate & $25 / 26(96.15 \%)$ & $25 / 27(92.59 \%)$ & $X^{2}=0.315$ & $1.0 \mathrm{a}$ \\
\hline
\end{tabular}

* significant difference, a Fisher's exact test.

Table II: Functional outcome.

\begin{tabular}{|c|c|c|c|}
\hline Functional outcome & $\begin{array}{c}\text { IMN } \\
(n=26)\end{array}$ & $\begin{array}{l}\text { Plating } \\
(n=27)\end{array}$ & Values \\
\hline VAS & & & $\begin{array}{c}f_{1}=1.518, p_{1}=0.224 \\
f_{2}=331.068, p_{2}<0.001\end{array}$ \\
\hline At 6 weeks & $4.96 \pm 1.11$ & $4.92 \pm 0.78$ & \\
\hline At 3 months & $3.46 \pm 0.81$ & $3.00 \pm 0.67$ & \\
\hline At 6 months & $1.73 \pm 0.82$ & $1.59 \pm 0.84$ & \\
\hline At 12 Months & $0.88 \pm 0.99$ & $0.74 \pm 0.71$ & \\
\hline ADL & & & $\begin{array}{l}f_{1}=3.545, p_{1}=0.065 \\
f_{2}=577.68, p_{2}<.001^{*}\end{array}$ \\
\hline At 6 weeks & $17.50 \pm 1.92$ & $18.51 \pm 2.08$ & \\
\hline At 3 months & $23.26 \pm 1.77$ & $23.11 \pm 1.33$ & \\
\hline At 6 months & $25.88 \pm 1.42$ & $26.66 \pm 1.35$ & \\
\hline At 12 Months & $28.00 \pm 1.35$ & $28.51 \pm 1.01$ & \\
\hline ASES & & 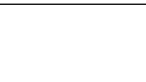 & $\begin{array}{c}f_{1}=3.145, p_{1}=0.082 \\
f_{2}=706.88, p_{2}<.001^{*}\end{array}$ \\
\hline At 6 weeks & $54.68 \pm 5.37$ & $56.23 \pm 5.09$ & \\
\hline At 3 months & $71.47 \pm 5.10$ & $73.51 \pm 4.22$ & \\
\hline At 6 months & $84.48 \pm 5.53$ & $86.48 \pm 5.85$ & \\
\hline At 12 months & $92.24 \pm 6.71$ & $93.82 \pm 4.70$ & \\
\hline
\end{tabular}

(Repeated measures ANOVA), Comparison of mean VAS, ADL and ASES score between the two group at each follow up- $f_{1}$ and $p_{1}$, and between the subsequent follow-ups in both group $-f_{2}$ and $p_{2}\left({ }^{*}=\right.$ significant difference, $\left.p<0.05\right)$.

Table III: Comparison of postoperative complications.

\begin{tabular}{lccc}
\hline Complication & IMN & Plate & p-value \\
& $\mathrm{n}(\%)$ & $\mathrm{n}(\%)$ & \\
\hline Total number of overall complications & $6 / 26(23.1)$ & $14 / 28(50)$ & $0.041 \mathrm{a}$ \\
Implant failure & $0 / 26$ & $1 / 28(3.6)$ & 1.0 \\
latrogenic radial nerve palsy (transient) & $0 / 26$ & $2 / 28(7.1)$ & 0.491 \\
Infection & $0 / 26$ & $2 / 27(7.4)$ & 0.491 \\
Delayed union & $3 / 26(11.5)$ & $6 / 27(22.2)$ & 0.467 \\
Non-Union & $1 / 26(3.8)$ & $2 / 27(7.4)$ & 1.0 \\
$\quad$ Restriction of joint movement: Shoulder & $2 / 26(7.7)$ & $1 / 27(3.7)$ & 0.610 \\
\hline Reoperations due to complications: & $1 / 26(3.8)$ & $3 / 28(10.7)$ & 0.612 \\
\hline a Chi square test ( $p=0.041$, Significant difference); & ${ }^{*}$ Fisher's exact test. &
\end{tabular}

indicating the increase in the number of male patients in both groups, but no statistically significant difference was found when compared $(p=0.712)$. No statistically significant difference was found between the two groups regarding $\mathrm{MOI}$, affected side, fracture type; open or closed, fracture classification and position ( $p>0.05)$.

The intraoperative blood loss, operative time, duration of hospital stay were significantly lower $(p<.001)$ in the IMN group than in the plate group. The fracture healed significantly earlier in the IMN group compared to those in the plating group $(p=0.022)$ (Table I). However, there was no significant difference in the union rate while comparing between the two groups $(p=1.0$, Table I).

No significant difference was noted when the mean VAS, $A D L$ and ASES scores were compared between the two groups at each follow-up (p1>0.05) (Table II). However, when these were compared between subsequent followups from 6 weeks to 12 weeks, a significant increase indicating the improvement in functional outcome was observed in both groups (p2<.001, Table II).

Individually, except restriction of shoulder joint function, the incidence of other complications was found to be higher in the plating group without a statistically significant difference. However, the total number of overall complications was significantly lower in the IMN group $(p=0.041$, Table III).

\section{DISCUSSION}

Humeral shaft fracture is more common in men, and RTA is the most common mechanism of injury. ${ }^{12} \mathrm{~A}$ similar result was found in this study. The closed, middle third of the humerus with AO/OTA type 12A was the most common type of injury noticed in both groups in this study, which is comparable with previous study. ${ }^{13}$ No statistically significant difference was observed when the preoperative symptom time was compared between the two groups since open fractures were emergencies, and the rest were handled as elective cases until the patients were fit for surgery.

Fixation with IMN is a much simpler technique than plating as there was minimal exposure ${ }^{14}$, which results in shorter operative time and less intraoperative blood loss. ${ }^{15}$ Due to its minimal invasive procedure and its simpler technique, the length of hospital stay after definitive management in IMN group was also significantly shorter than in the plating group. ${ }^{9} \mathrm{~A}$ similar result was established in this study.

An earlier union time with a significant difference after plating was mentioned. ${ }^{16}$ On the contrary, Fan et al. and others suggested a significantly shorter union time with IMN fixation.9,7,17 A similar result was observed in this study. A higher union rate of $96.15 \%$ was observed in IMN group as compared to $92.59 \%$ in plating group with an insignificant difference in this study, which matches 
with the findings from previous reports. ${ }^{18}$ Due to the minimally invasive procedure during IMN fixation with the prevention of fracture hematomas, soft tissues, blood vessels and periosteum around the fracture, it allows higher rates of union and good results as observed in our study. Literature has also shown that cases with a shorter healing time were those treated with closed reduction, ${ }^{11}$ as in the setting of IMN fixation which rarely requires open reduction.

In the study conducted by Putti et al., 19 there was no significant difference in the mean ASES functional scores between the 2 groups which were contrary to the findings of previous study. ${ }^{16}$ In the present study when we compared the mean ASES, the mean VAS and the mean ADL scores between the two groups at each follow-up, no significant difference was found. But when these were compared between the subsequent followups in both groups, a significant difference was noted, only to indicate a significant progressive improvement in function over a period of time in both groups. Bauze et al. has suggested a medial starting point that avoided the avascular area of the rotator cuff without compromising its healing, that can lead to a good functional outcome of the shoulder joint later on. ${ }^{20}$ In this study also the nail was inserted medially to the tip of the greater tuberosity, $0.5 \mathrm{~cm}$ posterior to the bicipital groove to minimise damage to the rotator cuff and also properly countersinking the tip of the nail was done.

Many studies in the past have highlighted numerous disadvantages of ORIF with plate fixation since it requires extensive open dissection which increases the risk of radial nerve damage, infection, delayed union and nonunion. 6

latrogenic radial nerve palsy was higher with $2 / 28$ cases $(7.1 \%)$ in the plating group and $0 / 26$ in the IMN group in this study, with an insignificant difference as in the previous study. 15 Those two cases were managed conservatively and recovered well in a mean time of two months indicating a neuropraxic type of injury.

Infection was also higher with 2/27 cases of superficial infection in the plating group, also without a significant difference as in earlier studies. ${ }^{19}$ This may be explained by a wide open surgery, soft tissue and periosteal stripping from the bone, and the prolonged exposure with a longer operating time during plate fixation.6,21

In the previous reports on plating, the incidence of nonunion has ranged from $2 \%$ to $10 \% .{ }^{22}$ Retrospectives studies of IMN fixation reported lower incidence of nonunion ranging from 0 to $8 \% .{ }^{23}$ Higher nonunion after plating was observed in this study; which may again be explained by the preservation of the fracture site biology during IMN fixation which was not possible with plate fixation procedure. In contrary, plate fixation showed fewer non-unions than IMN in other studies. ${ }^{11}$ The narrow medullary canal may cause jamming of the nail and contribute to distraction at the fracture site during nailing. 24 We disagree to this as appropriate reaming, and then carefully tapping the insertion handle attached to the tip of the nail with a bone hammer proximally after the nail insertion and the distal locking screw properly placed can counteract the distracting force and minimise the gap between the fracture fragments. Raghavendra et al. also found a higher incidence of delayed union with plating. ${ }^{13}$

Earlier in this study, the authors had found that there was no significant difference in the functional outcome of the shoulder between the two groups although slightly higher incidence of restriction of the shoulder joint movement after antegrade IMN fixation was established. A similar result was also found in previous studies. ${ }^{19}$ In this study, in IMN group, one case had the nail head found outside the humeral head with ASES score of 68.33 at 12 months; another one had near normal ROM with a VAS score of 2 . And in the plating group, one had frozen shoulder due to inadequate mobilisation. Impaired function of the shoulder joint with antegrade IMN may be a result of impingement due to proximal migration, rotator cuff violation, and adhesive capsulitis. ${ }^{23}$ When performed properly, as explained earlier with a medial starting point and countersinking the nail head, a minimal restriction was observed in this study with an insignificant difference between the two groups. Flinkkila et al. have also confirmed that ante-grade nailing, if performed correctly, is not responsible for the dysfunction of the shoulder joint. ${ }^{25}$

The present study revealed a higher incidence of implant failures with plating, without a significant difference; one with a broken plate seen in the follow-up. This case was reoperated with bone graft and plate fixation. This result is consistent with the previous study with a higher incidence of implant failure in plating. ${ }^{13}$ Implant failure in plating may be explained by the stress rising at the end of the plate, ${ }^{7}$ as well as the stress shielding of the bone by the plate at the fracture site which reduces the strength of the union and, it also provides less secure fixation especially in the osteopenic bone. 15

In the present study, a higher rate of reoperation was observed in plating group without a significant difference. This result was unlike the study conducted by Kumar et al., ${ }^{1}$ where IMN had an increased rate of reoperations, also without a statistically significant difference. Three cases in the plating group (one implant failure and two nonunion) and one case of nonunion in IMN, were reoperated and fixed with a plate and bone graft in this study.

Kulkarni et al. concluded that the overall complication rate was higher in the IMN group without a significant difference in their study. ${ }^{15}$ But, overall significantly higher total complication rate in the plating group was observed in this study. 


\section{CONCLUSION}

The application of the IMN was simple and minimally invasive that preserved the biology of the fracture site, which resulted in a significant decrease in intra-operative blood loss, shorter operation time, shorter hospital stays, union times, and a lower rate of overall complications. Despite no significant difference between the two methods in terms of functional outcome and union rate of the fracture, significant progressive improvement in the functional outcome over a course of time was assured with both methods. The patients treated with plating underwent more reoperations with secondary bone graft procedures in this study. Therefore, IMN may be a better choice of internal fixation, as it also accelerates the patient's recovery and increases their satisfaction.

\section{ETHICAL APPROVAL:}

Our study was approved by the Ethics Committee of Institute of the $1^{\text {st }}$ Affiliated Hospital of Jinzhou Medical Univeristy of China.

\section{PATIENTS' CONSENT:}

Informed consents were obtained from all participants.

\section{CONFLICT OF INTEREST:}

Authors declared no conflict of interest.

\section{AUTHORS' CONTRIBUTION:}

YW, YK: The conception and design of the work; the acquisition, analysis, interpertation of data.

YC: Needful corrections, advices and final approval.

ZG, YY, YB: Provided ideas on the status, and reviewed the paper.

\section{FUNDING:}

This work was supported by National Natural Science Foundation of China (NSFC) under grant No.81501659, 81501959, 81571890, and 81671907. Project supported by the Natural Science Foundation of Science and Technology Department of Liaoning province under grant No.201602323. The project is sponsored by Liaoning BaiQianWan Talents Program.

\section{REFERENCES}

1. Kumar R, Singh P, Chaudhary LJ, Singh S. Humeral shaft fracture management, a prospective study; nailing or plating. J Clin Orthop Trauma 2012; 3:37-42.

2. Klenerman L. Fracture of the shaft of the humerus. J Bone $J t$ Surg $\mathrm{Br}$ 1966; 48:105-11.

3. Cole PA, Wijdicks CA. The operative treatment of diaphyseal humeral shaft fractures. Hand Clin 2007; 23:437-48.

4. Hashmi PM, Mohib Y, Abbas K. Functional and radiological outcome of Intramedullary nailing $v s$. plate fixation for humeral shaft fractures. Does treatment method bring any difference? J Pak Med Assoc 2014; 64(12 Suppl 2):S135-8.
5. Zarkadis NJ, Eisenstein ED, Kusnezov NA, Dunn JC, Blair JA. Open reduction-internal fixation versus intramedullary nailing for humeral shaft fractures: An expected value decision analysis. J Shoulder Elb Surg 2018; 2018 ;27:204-10.

6. Ring D, Jupiter JB. Internal fixation of the humerus with locking compression plates. Tech Shoulder Elb Surg 2003; 4:169-74.

7. Changulani M, Jain UK, Keswani T. Comparison of the use of the humerus intramedullary nail and dynamic compression plate for the management of diaphyseal fractures of the humerus. A randomised controlled study. Int Orthop 2007; 31391-5.

8. Saleem Khan A, Afzal W, Anwar A. Comparison of shoulder function, radial nerve palsy and infection after nailing versus plating in humeral shaft fractures. J Coll Physicians Surg Pakistan 2010; 20:253-7.

9. Fan Y, Li Y-W, Zhang H-B, Liu J-F, Han X-M, Chang X, et al. Management of humeral shaft fractures with intramedullary interlocking nail versus locking compression plate. Orthopedics 2015; 38:e825-9.

10. Denies E, Nijs S, Sermon A, Broos P. Operative treatment of humeral shaft fractures: Comparison of plating and intramedullary nailing. Acta Orthop Belg 2010; 76:735-42.

11. Kesemenli CC, Subasi M, Arslan H, Necmioglu S, Kapukaya A. Comparison between the results of intramedullary nailing and compression plate fixation in the treatment of humerus fractures. Acta Orthop Traumatol Turc 2003; 37:120-5.

12. Tsai $\mathrm{CH}$, Fong $\mathrm{YC}$, Chen $\mathrm{YH}, \mathrm{Hsu} \mathrm{CJ}$, Chang $\mathrm{CH}$, Hsu HC. The epidemiology of traumatic humeral shaft fractures in Taiwan. Int Orthop 2009; 33:463-7.

13. Raghavendra S, Bhalodiya HP. Internal fixation of fractures of the shaft of the humerus by dynamic compression plate or intramedullary nail: A prospective study. Indian J Orthop 2007; 41:214-8.

14. Lin J, Shen PW, Hou SM. Complications of locked nailing in humeral shaft fractures. J Trauma 2003; 4:943-9.

15. Kulkarni SG, Varshneya A, Jain M, Kulkarni VS, Kulkarni GS, Kulkarni MG, et al. Antegrade interlocking nailing versus dynamic compression plating for humeral shaft fractures. J Orthop Surg (Hong Kong) 2012; 20:288-91.

16. Singisetti K, Ambedkar M. Nailing versus plating in humerus shaft fractures: A prospective comparative study. Int Orthop 2010; 34:571-6.

17. Kulkarni VS, Kulkarni MS, Kulkarni GS, Goyal V, Kulkarni MG. Comparison between antegrade intramedullary nailing (IMN), open reduction plate osteosynthesis (ORPO) and minimally invasive plate osteosynthesis (MIPO) in treatment of humerus diaphyseal fractures. Injury 2017; 48 (Suppl 2):S8-S13.

18. An Z, Zeng B, He X, Chen Q, Hu S. Plating osteosynthesis of middistal humeral shaft fractures: Minimally invasive versus conventional open reduction technique. Int Orthop 2010; 34:131-5.

19. Putti $A B$, Uppin RB, Putti BB. Locked intramedullary nailing versus dynamic compression plating for humeral shaft fractures. J Orthop Surg 2009; 17:139-41.

20. Bauze A, Clayer M. Treatment of pathological fractures of the humerus with a locked intramedullary nail. J Orthop Surg 2003; 11:34-7.

21. Gallucci GL, Boretto JG, Alfie VA, Donndorff A, De Carli P. Posterior minimally invasive plate osteosynthesis (MIPO) of 
distal third humeral shaft fractures with segmental isolation of the radial nerve. Chir Main 2015; 34:221-6.

22. Heim D, Herkert F, Hess P, Regazzoni P. Surgical treatment of humeral shaft fractures - the basel experience. J Trauma - Inj Infect Crit Care 1993; 35:226-32.

23. McCormack RG, Brien D, Buckley RE, McKee MD, Powell J, Schemitsch EH. Fixation of fractures of the shaft of the humerus by dynamic compression plate or intramedullary nail. A pros- pective, randomised trial. J Bone Joint Surg Br 2000; 82:336-9.

24. Hems TEJ, Bhullar TPS. Interlocking nailing of humeral shaft fractures: The Oxford experience 1991 to 1994. Injury 1996; 27:485-9.

25. Flinkkilä $T$, Hyvönen $P$, Siira $P$, Hämäläinen $M$. Recovery of shoulder joint function after humeral shaft fracture: A comparative study between antegrade intramedullary nailing and plate fixation. Arch Orthop Trauma Surg 2004; 124:537-41. 